\title{
Oscillations in Endogenous Inputs to Neurons Affect Excitability and Signal Processing
}

\author{
Marjorie A. Parkis, ${ }^{2}$ Jack L. Feldman, ${ }^{3}$ Dean M. Robinson, ${ }^{1}$ and Gregory D. Funk ${ }^{1,4}$ \\ ${ }^{1}$ Department of Physiology, Faculty of Medical and Health Sciences, University of Auckland, Auckland, New Zealand, ${ }^{2}$ Department of Organismal Biology \\ and Anatomy, University of Chicago, Chicago, Illinois 60637, ${ }^{3}$ Systems Neurobiology Laboratory, Department of Neurobiology, University of California, Los \\ Angeles, Los Angeles, California 90095-1763, and ${ }^{4}$ Department of Physiology, Faculty of Medicine and Dentistry, University of Alberta, Edmonton, Alberta, \\ T6G 2H7, Canada
}

Synchrony and oscillations in neuronal firing play important roles in information processing in the mammalian brain. Here, we evaluate their role in controlling neuronal output in a well defined motor behavior, breathing, using an in vitro preparation from neonatal rat that generates respiratory-related motor output. In this preparation, phrenic motoneurons (PMNs) receive endogenous rhythmic inspiratory currents with prominent oscillations in the $20-50 \mathrm{~Hz}$ range. We recorded these inspiratory currents in individual PMNs and used them as test inputs for the same motoneuron (MN) during the normally silent expiratory periods. The impact of the oscillations on $\mathrm{MN}$ output was evaluated by filtering the currents before injection. Responses to unfiltered inspiratory currents were indistinguishable from voltage changes during spontaneous inspiratory periods. More than $90 \%$ of action potentials occurred within milliseconds [ -2 to +4$]$ of the oscillation peaks. The timing of action potentials was highly reproducible in response to unfiltered currents. Attenuation of the oscillations by low-pass filtering $(<50 \mathrm{~Hz})$ decreased the precision in action potential timing and significantly reduced the number of action potentials by $\sim 35 \%$. The adrenergic agonist phenylephrine increased instantaneous firing frequency in responses evoked by squarewave or low-pass filtered inspiratory currents but had no effect on firing frequency evoked by unfiltered currents. We conclude that oscillations control the precise timing of action potentials, help to maximize synaptic drive efficiency, and constrain MN firing frequencies to those optimal for muscle contraction.

Key words: oscillation; phrenic motoneuron; phenylephrine; respiration; excitability; rat; whole-cell recording

\section{Introduction}

Determining how synaptic inputs are transformed into patterns of action potential output is key to understanding information processing in the CNS. The role of intrinsic membrane properties in this transformation is illuminated by approaches that use tonic afferent stimulation or intracellular injection of square-wave or ramp current waveforms to activate neurons. The dynamic characteristics of endogenous synaptic inputs to neurons that are important in transforming input into output are less well characterized. A prominent dynamic component of endogenous inputs is high-frequency $(>10 \mathrm{~Hz})$ oscillations. These oscillations can be seen throughout the CNS (Gray, 1994; Fetz et al., 2000), including in networks that provide rhythmic excitatory drive to motoneurons (MNs) in behaviors such as chewing (Smith and Denny, 1990), speaking (Smith and Denny, 1990; Nakazawa et al., 2000), and breathing (Cohen and Gootman, 1969; Cohen et

Received Feb. 12, 2003; revised May 22, 2003; accepted June 30, 2003.

This work was supported by grants from the Marsden Fund, Health Research Council of New Zealand, Lotteries Health, Auckland Medical Research Foundation, New Zealand Neurological Foundation, Maurice and Phyllis Paykel Trust, and National Institutes of Health Grant NS 24742. We thank M. Glasson and M. Navakatykyan for technical assistance.

Correspondence should be addressed to Gregory D. Funk, Department of Physiology, Faculty of Medicine and Dentistry, University of Alberta, 7-55 Medical Science Building, 87 Avenue, 114 Street, Edmonton, Alberta, T6G 2H7, Canada. E-mail: gf@ualberta.ca.

Copyright $\odot 2003$ Society for Neuroscience $\quad$ 0270-6474/03/238152-07\$15.00/0 al., 1987; Liu et al., 1990; Smith and Denny, 1990; Christakos et al., 1991; Tarasiuk and Sica, 1997; Funk and Parkis, 2002). Oscillations with peak power in the $20-150 \mathrm{~Hz}$ range are ubiquitous in the respiratory network. They are synchronized between different network elements (Liu et al., 1990; Cohen et al., 1997; Tarasiuk and Sica, 1997), yet their function is not known. Here, we explore the importance of endogenous oscillations in synaptic input for $\mathrm{MN}$ information processing. We recorded endogenous inspiratory currents in phrenic MNs (PMNs) in a neonatal rat brainstem spinal cord preparation that spontaneously generates rhythmic, respiratory-related motor nerve output (Smith and Feldman, 1987). These endogenous currents were reinjected as command inputs, before and after low-pass filtering, into the same PMN during the normally quiescent expiratory period, and the voltage response was recorded. We also compared the effects of phenylephrine (PE), a potent neuromodulator agonist, on the voltage response of PMNs to these filtered and unfiltered inspiratory currents, as well as to traditional square-wave currents. Analysis of MNs offers the advantage that, as the final common output path from the CNS to skeletal muscles, the physiological significance of changes in their input-output relationships is readily interpretable.

\section{Materials and Methods}

Preparation. Brainstem-spinal cords were isolated from neonatal Wistar rats ranging in age from postnatal days $0-3$ as described previously 
(Smith and Feldman, 1987; Parkis et al., 2000). Preparations were pinned down, ventral surface up, on Sylgard resin in a recording chamber $(2 \mathrm{ml}$ volume) and perfused at a flow rate of $2-4 \mathrm{ml} / \mathrm{min}$ with oxygenated artificial CSF (in mM): $120 \mathrm{NaCl}, 3 \mathrm{KCl}, 1 \mathrm{CaCl}_{2}, 2 \mathrm{MgSO}_{2}, 26 \mathrm{NaHCO}_{3}$, $1.25 \mathrm{NaH}_{2} \mathrm{PO}_{4}$, and 20 D-glucose, $\mathrm{pH} 7.45$ (oxygenated with $95 \% \mathrm{O}_{2}-5 \%$ $\mathrm{CO}_{2}$ at $\left.26-27^{\circ} \mathrm{C}\right)$.

Recording. Population inspiratory activity was recorded from the severed ends of $\mathrm{C} 1$ or $\mathrm{C} 4$ nerve roots using suction electrodes, amplified, filtered $(0.3-3 \mathrm{kHz})$, rectified, and integrated (Smith and Feldman, 1987; Parkis et al., 2000). Whole-cell voltage- and current-clamp data were recorded from PMNs using an Axopatch 1D amplifier (Axon Instruments, Foster City, CA) controlled by Axodata version 1.2.2 (Axon Instruments) and custom-written programs developed in LabVIEW (Parkis et al., 2000). Intracellular recording electrodes (3.5-4.5 $\Omega$ ) contained the following (in $\mathrm{mm}$ ): $125 \mathrm{~K}^{+}$-gluconate, $5 \mathrm{NaCl}, 1 \mathrm{CaCl}_{2}, 10$ HEPES, 10 BAPTA, and $2 \mathrm{Mg}^{2+}$-ATP, $\mathrm{pH}$ 7.2-7.3. All signals were recorded on videotape via PCM digital processor (Vetter 3000A; Vetter Instruments, Reberburg, PA).

Protocols. A software program developed in our laboratory using the LabVIEW (National Instruments, Austin, TX) programming environment was used to activate neurons with endogenous waveforms of synaptic current (Parkis et al., 2000). Voltage-clamp recordings of individual inspiratory synaptic currents were triggered from bursts of inspiratory activity on $\mathrm{C} 1$ or $\mathrm{C} 4$ ventral nerve, and 5-10 different synaptic waveforms were acquired $(20 \mathrm{kHz})$ to a waveform library specific for that cell. The recording configuration was then switched to current clamp, and a waveform was selected to be used as a test input. Because of the negative sign of inward currents measured in voltage clamp, the recorded synaptic waveform was inverted by the LabVIEW program so that injected inspiratory currents would be depolarizing. Each motoneuron was activated with one of its own recorded currents.

When currents were filtered before injection, a low-pass Butterworth filter (order of 3-5) was used. Different waveforms (representing the same initial recorded current filtered using different cutoff frequencies) were presented a minimum of four times in random order, and the number of spikes produced by each stimulus was reported as an average of four responses. Unfiltered waveforms were presented twice as often as other stimuli to ensure consistent control responses. Current injections were triggered to occur in the quiescent (expiratory) period between inspiratory bursts.

To examine the effects of $\alpha 1$ noradrenergic receptor activation on responses of PMNs to injection of smooth and synaptic current waveforms, the $\alpha 1$ receptor agonist PE was locally applied (5-10 min) via a pressure injection pipette over the phrenic nucleus (Parkis et al., 1999). Control and PE responses were elicited from the same membrane potential by injecting DC current to correct for the PE-induced depolarization. To control for time- and activity-dependent changes in firing output that differ between different waveforms, analysis of all responses was limited to the time range over which PMNs fired in response to stimulation with unfiltered waveforms (see Fig. $3 a, b$, dashed boxes). The magnitude of the square-wave current pulse was selected to produce an average instantaneous firing rate similar to that produced by endogenous unfiltered synaptic currents in control. Maximum effects of PE on C4 nerve inspiratory activity for each animal were taken to be the maximum value in a moving average of five consecutive bursts within 2 min of PE application.

Analysis. Offline analysis was performed using AxoGraph (version 4.3) and Microsoft (Seattle, WA) Excel (version 5.0) software. Power spectra of captured currents were generated offline using LabVIEW software. Differences between means were compared using one-way ANOVA (SAS 6.1; SAS Institute, Cary, NC). Linear contrast coefficients were used to partition the sum of squares to determine significant differences between groups. Values of $p<0.05$ were considered significant. Data are given as mean \pm SE.

\section{Results}

\section{Characteristics of inspiratory currents}

Experiments were performed in a neonatal rat brainstem-spinal cord preparation (Smith and Feldman, 1987) that generates endogenous, periodic inspiratory-related synaptic inputs to PMNs
A
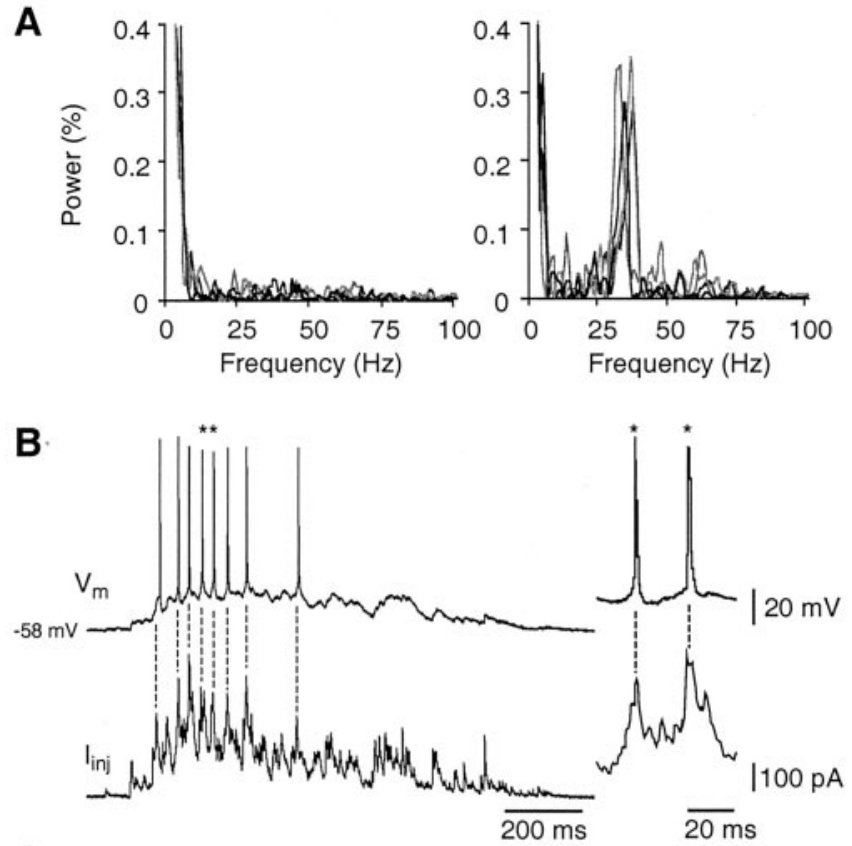

C

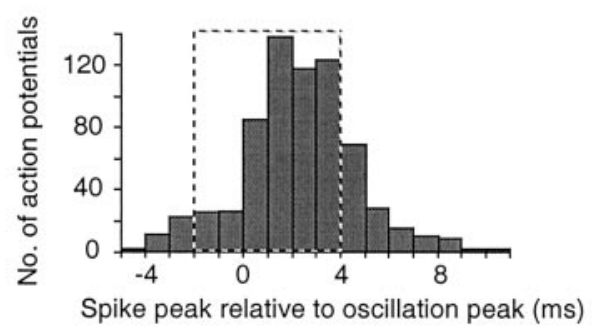

Figure 1. Action potentials arise preferentially on peaks of high-frequency oscillations. A, Power spectra of four consecutive inspiratory synaptic currents from two different PMNs, receiving inspiratory drive currents with different amounts of oscillatory activity. $B$, Stimulation of a PMN with an inspiratory synaptic current waveform (bottom trace) elicited membrane depolarization and eight action potentials. Dashed lines highlight close correlation between peaks of oscillations in the injected current and occurrence of action potentials. Asterisks in $B$ (left) denote action potentials shown in expanded time scale to the right. C, Histogram showing temporal distribution of 689 action potentials recorded in 13 PMNs relative to nearest peak in the injected inspiratory waveform. Time 0 indicates an oscillation peak. Dashed box indicates the 6 msec interval during which $92 \%$ of action potentials occurred.

(Liu et al., 1990; Rekling et al., 2000a). These currents were synchronous with bursts of inspiratory activity on the $\mathrm{C} 4$ spinal cord ventral nerve root, had peak amplitudes of $-803 \pm 55 \mathrm{pA}(n=$ $35)$, and exhibited a rapidly increasing (140 $\pm 10 \mathrm{msec}$ to peak) slowly decrementing $(530 \pm 30 \mathrm{msec})$ waveform.

Power spectral analysis of the inspiratory drive currents to PMNs ( $n=22$ neurons; $5-17$ consecutive cycles per neuron) revealed two main patterns of synaptic input. Six PMNs received synaptic currents that contained only small-amplitude peaks in the $20-50 \mathrm{~Hz}$ range $(\sim 0.04 \%$ of total power $)$, centered at $32 \pm$ $1.7 \mathrm{~Hz}$ (Fig. $1 A$, left). Sixteen PMNs received inspiratory synaptic inputs that contained significant peaks at $32 \pm 1.4 \mathrm{~Hz}$ with mean peak power more than threefold higher $(0.13 \pm 0.01 \%$ of total power) (Fig. $1 A$, right). These MNs were otherwise indistinguishable on the basis of input resistance, rheobase, membrane capacitance, or magnitude of synaptic current.

\section{Oscillations control action potential timing}

To reproduce as closely as possible the dynamic pattern of membrane depolarization that occurs during actual respiratory behav- 


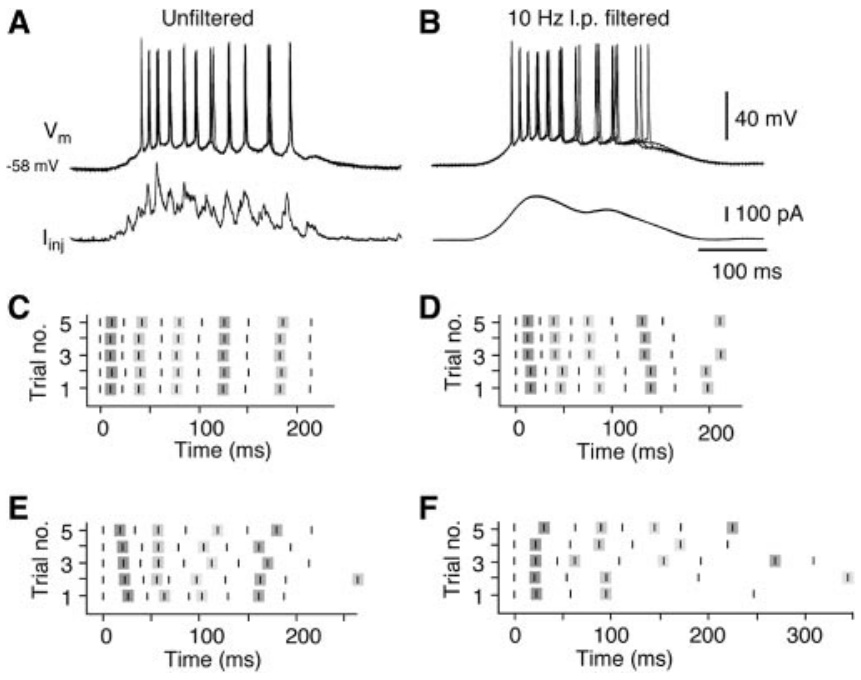

Figure 2. Oscillations on inspiratory synaptic currents increase precision of action potential timing during repetitive firing. Membrane potential responses (top traces) of a PMN to repeated application of an inspiratory synaptic current waveform $\left(I_{\text {inj }}\right)$ in its unfiltered $(A)$ and filtered $(B$; $10 \mathrm{~Hz}$ low pass) form. Membrane potential responses are shown superimposed (first 4 trials). $C$, $D$, Raster plots of spike times relative to the onset of the first action potential in the train (5 consecutive trials). $E, F$, Raster plots showing spike times relative to the onset of the first action potential in five consecutive endogenous inspiratory cycles. The plot in $E$ represents activity of a PMN driven by inputs with consistent oscillations. Synaptic inputs to the MN in F lacked oscillations.

ior, we used our method for repeatedly stimulating each PMN with an inspiratory synaptic current waveform recorded from that MN (Parkis et al., 2000). These endogenous inspiratory current waveforms were injected during the quiescent period between endogenous inspiratory bursts, i.e., during the expiratory period. The membrane depolarization evoked by spontaneous inspiratory inputs, averaged from a representative sample of PMNs that received consistent inspiratory inputs (four consecutive spontaneous inspiratory cycles from four different PMNs $(26.5 \pm 3.5 \mathrm{mV})$, was the same as that evoked in the same MNs by reinjected synaptic currents $(29.2 \pm 4.7 \mathrm{mV})$.

Consistent with responses of hypoglossal MNs to simulated postsynaptic currents of varying amplitude (Türker and Powers, 1999), the timing of action potentials and the occurrence of oscillations in the endogenous current waveform were closely correlated (Fig. 1 B). Most action potentials (92\%; 631 of $689 ; 124$ current injections in 13 PMNs) occurred within a $6 \mathrm{msec}$ window around the oscillation peaks $(-2$ to $+4 \mathrm{msec})$ in which the average interpeak interval was $\sim 32 \mathrm{msec}$ (Fig. $1 C$ ).

We then tested the hypothesis, on the basis of responses of cortical neurons to random white noise inputs (Mainen and Sejnowski, 1995), that synaptically generated oscillations increase the temporal precision, i.e., reproducibility of action potential occurrence during repetitive firing. PMNs were stimulated repeatedly with the same synaptic current waveforms before and after low-pass filtering at $10 \mathrm{~Hz}$. Repeated stimulation with unfiltered currents produced highly consistent spike trains (Fig. 2). In six PMNs tested in this manner, the SDs of the delay between first and second, and first and last action potentials averaged across all cells were $0.67 \pm 0.20$ and $1.80 \pm 0.54 \mathrm{msec}(n=6)$ (total burst duration of $190 \pm 24 \mathrm{msec}$ ), respectively. After filtering of the injected current, although significant clustering of action potentials was still apparent (reflecting the contribution of intrinsic membrane properties to this phenomenon), there was a significant reduction in the precision of action potential timing.
The second action potential of each train remained tightly locked to the first ( $\mathrm{SD}, 0.96 \pm 0.25 \mathrm{msec} ; n=6$ ). However, the timing of the last spike was highly variable $(\mathrm{SD}, 10.08 \pm 4.49 \mathrm{msec} ; n=6)$. The increased variability was also evident in the coefficient of variation for the delay between first and last spikes, which was sevenfold greater for responses to filtered $(0.063 \pm 0.025)$ compared with unfiltered $(0.009 \pm 0.002)$ waveforms. The coefficient of variation for the last interspike interval of responses to filtered currents $(0.118 \pm 0.037 ; n=6)$ was also significantly increased over that for unfiltered waveforms $(0.022 \pm 0.009 ; n=6)$. These data suggest that naturally occurring oscillations in synaptic drive play a critical role in determining the timing of action potentials during repetitive firing.

One possible function for these oscillations is to ensure optimal motor unit activation by producing a precise pattern of output in each motoneuron. If so, one would predict that the inspiratory discharge patterns of MNs receiving synaptic inputs with strong oscillations would be more consistent than those without. To test this possibility, we measured the variability in spike timing of bursts generated by consecutive spontaneous currents in four PMNs receiving inputs with prominent oscillations and four different PMNs receiving inputs that lacked prominent oscillations. Compared with PMNs receiving inputs that lacked oscillations (Fig. 2 F), discharge patterns of PMNs receiving inputs with consistent oscillations showed much less variability for consecutive spontaneous bursts (Fig. 2 E). The average coefficient of variation for the interval between the first and seventh spikes was twofold higher in the MNs driven by inputs that lacked oscillations $(0.16 \pm 0.10)$ compared with those with strong oscillations $(0.08 \pm 0.01)$. Similarly, the coefficients of variation for the last interspike interval for MNs with and without strong oscillations were $0.27 \pm 0.11$ and $0.41 \pm 0.07$, respectively. Thus, although variations in the pattern of synaptic input to individual MNs mean that the pattern of action potential output will not be identical between consecutive cycles, oscillations in the input reduce variability in interspike interval as predicted, and play an important role in controlling spike timing.

\section{Oscillations constrain mechanisms by which modulators alter excitability}

Neuromodulators such as norepinephrine, substance P, and thyrotropin-releasing hormone increase MN excitability (Rekling et al., 2000a; Powers and Binder, 2001) by reducing a passive resting $\mathrm{K}^{+}$leak conductance and by inhibiting active conductances, such as the $\mathrm{Ca}^{2+}$-activated $\mathrm{K}^{+}$conductance. When the impact of these modulators on $\mathrm{MN}$ firing behavior is tested using square-wave inputs, these effects produce a leftward shift and an increase in the slope of the relationship between firing frequency and current. The net result for MN output is higher discharge frequencies and, for nonsquare-wave inputs, earlier onset of firing. In other words, one would expect an excitatory modulator to significantly alter the frequency and number of action potentials evoked by a given input waveform.

If, however, oscillations preserve action potential timing under conditions in which excitability is markedly changed, we would expect neuromodulators to have less impact on firing frequency when $\mathrm{MNs}$ receive inputs with oscillations. We tested this by comparing the effects of PE on instantaneous firing frequency produced by reinjected, unfiltered, inspiratory waveforms with that produced by smooth waveforms (filtered inspiratory waveforms and square-wave forms). We also assessed the effects of PE on the firing frequency evoked in PMNs by spontaneous inspiratory inputs. 

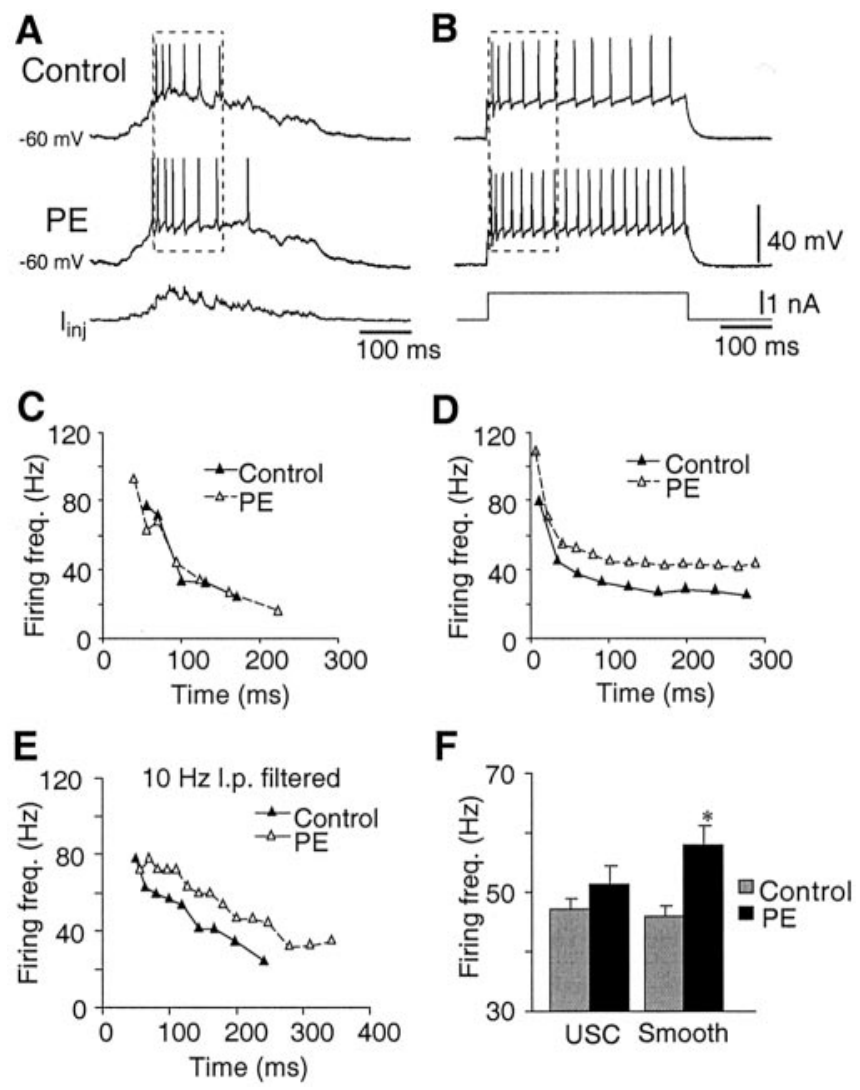

Figure 3. PE induced increases in instantaneous firing frequency responses to smooth, but not endogenous, input waveforms. Membrane potential responses of a PMN to injection of an inspiratory synaptic waveform $(A)$ and a square-wave current pulse $(B)$ in the absence (control) and presence of $\mathrm{PE}(100 \mu \mathrm{m}) . C, D$, Instantaneous firing frequencies for the responses evoked in $A$ and $B$ are plotted versus time in C and $D$, respectively. $E$, Effect of $P E$ on the response of a PMN to stimulation with a $10 \mathrm{~Hz}$ low-pass filtered inspiratory synaptic waveform. F, Effects of PE on instantaneous firing frequency evoked through unfiltered synaptic currents (USC) and smooth (square-wave or $10 \mathrm{~Hz}$ filtered) waveforms $(n=6)$ ) (dashed boxes in $A$ and $B$ indicate period over which firing frequency was analyzed). Asterisk denotes significant difference in firing frequency relative to control conditions $(p=0.008)$ and that magnitude of the increase in response to smoothed inputs was greater than that in response to USC ( $p=0.01)$.

Consistent with its effects on other MNs (Parkis et al., 1995; Binder et al., 1996; Rekling et al., 2000a), PE depolarized PMNs $(6.0 \pm 1.0 \mathrm{mV} ; n=6)$, decreased $I_{\text {rheo }}$ (from $540 \pm 96$ to $330 \pm 80$ $\mathrm{pA} ; n=5)$, and increased input resistance $\left(R_{\mathrm{n}}\right)(7-41 \%)$. In response to square-wave (Fig. $3 B, D$ ) and filtered (Fig. $3 E$ ) currents, PE significantly increased PMN instantaneous firing frequency from $46.0 \pm 2.1$ to $58.0 \pm 3.8 \mathrm{~Hz}(n=6)$ (Fig. $3 F)$. In contrast, when the same PMNs were activated with unfiltered inspiratory synaptic currents, PE did not affect instantaneous firing frequency (Fig. 3A,C,F). Instead, its main effect was to double the duration over which firing occurred, from $110 \pm 30$ to $220 \pm 40 \mathrm{msec}(n=6)$. This can be seen for one PMN in Figure $3 A$, in which the six action potentials evoked under control conditions occurred at similar time points in the presence of PE, i.e., they arose off the same peaks in the injected current. In PE, however, action potentials seen in control conditions were preceded and followed by additional action potentials that arose from peaks in the input waveform that previously produced subthreshold voltage deflections.

Similar behavior was apparent in response to spontaneous inspiratory inputs. PE had no effect on instantaneous firing frequency evoked by spontaneous inspiratory currents, regardless of
A

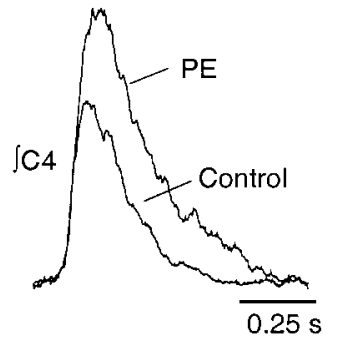

B

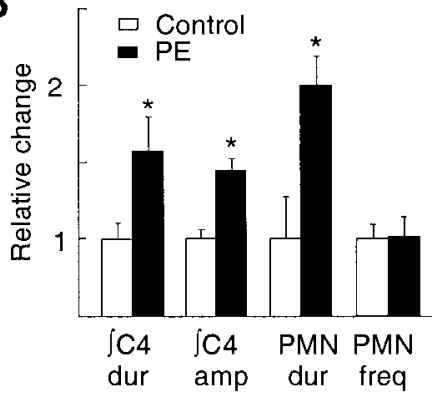

Figure 4. PE increases both the duration and amplitude of inspiratory bursts recorded from the (4 ventral nerve root (which contains the phrenic nerve) but not firing frequency of PMNs during inspiration. $A$, Inspiratory burst envelopes averaged from 10 consecutive inspiratory cycles $\left(\int(4)\right.$ before and during application of $\mathrm{PE}(100 \mu \mathrm{M})$. B, Maximum effects of PE on (4 nerve burst duration ( $\int\left(4\right.$ dur) and amplitude $\left(\int C 4 \mathrm{amp}\right.$ ) relative to control (control values, 1.0). Effects of PE on the average duration over which individual PMNs produced action potential output (PMN dur) in response to endogenous input is included for comparison. Note that the instantaneous firing frequency (PMN freq) evoked by endogenous inputs was not affected by PE.

whether or not the depolarization induced by PE was offset with DC current injection (control, $43.4 \pm 4.8 \mathrm{~Hz}, n=6$; PE with DC offset, $43.8 \pm 5.6 \mathrm{~Hz}, n=6$; PE without DC offset, $41.2 \pm 0.6 \mathrm{~Hz}$, $n=3)$. The duration of PMN discharge increased from $140 \pm 35$ msec in control to $250 \pm 71 \mathrm{msec}$ in PE $(n=6)$, which manifested as a significant increase in the duration $(57 \pm 22 \%)$ of inspiratory bursts recorded from the population of PMN axons contained in the C4 nerve root (Fig. 4). Because PE did not increase firing frequency, the PE-induced increase in integrated $\mathrm{C} 4$ nerve inspiratory burst amplitude $(45 \pm 7 \%)$ was likely attributable to increased duration of action potential firing and/or recruitment of additional PMNs.

\section{Oscillations increase input-output efficiency}

To further explore the importance of endogenous synaptically generated current oscillations in controlling repetitive firing behavior, we compared responses to unfiltered synaptic current waveforms and the same waveforms after low-pass filtering with cut-off frequencies between 10 and $100 \mathrm{~Hz}$. Low-pass filtering at $50 \mathrm{~Hz}$ and below reduced the number of action potentials (Fig. 5). Filtering at 50, 20-25, and $10 \mathrm{~Hz}$ reduced the number of action potentials elicited by injected currents to $91 \pm 4,74 \pm 5$, and $65 \pm$ $5 \%$, respectively, of the response to the unfiltered current (Fig. $5 C)(n=12)$. In the example of Figure 5 , the number of action potentials produced was reduced from six in control (unfiltered), to five with a $50 \mathrm{~Hz}$ cutoff, and to four with 25 and $10 \mathrm{~Hz}$ cutoff. This reduction in output corresponded to removal of a large peak in the power spectrum centered at $\sim 37 \mathrm{~Hz}$ (Fig. $5 B$ ). Integration of the areas under the injected current waveforms verified that filtering did not reduce the charge transferred by the injected current at any cut-off frequency (total charge transfer was reduced by $<0.1 \%$ at the lowest cut-off frequency of $10 \mathrm{~Hz}$ ). Reductions in total power by $2.1 \pm 0.7$ and $3.9 \pm 1.4 \%$ with lowpass filtering at 20 and $10 \mathrm{~Hz}$, respectively $(n=10)$, were also insignificant.

\section{Discussion}

Synchronized oscillations in neuronal activity with power in the range of 10-150 Hz appear throughout the brain (Gray, 1994) and are postulated to contribute to a variety of higher functions (Konig and Engel, 1995; Engel et al., 1999), including memory formation, control of arousal state, and "binding" of neuronal 

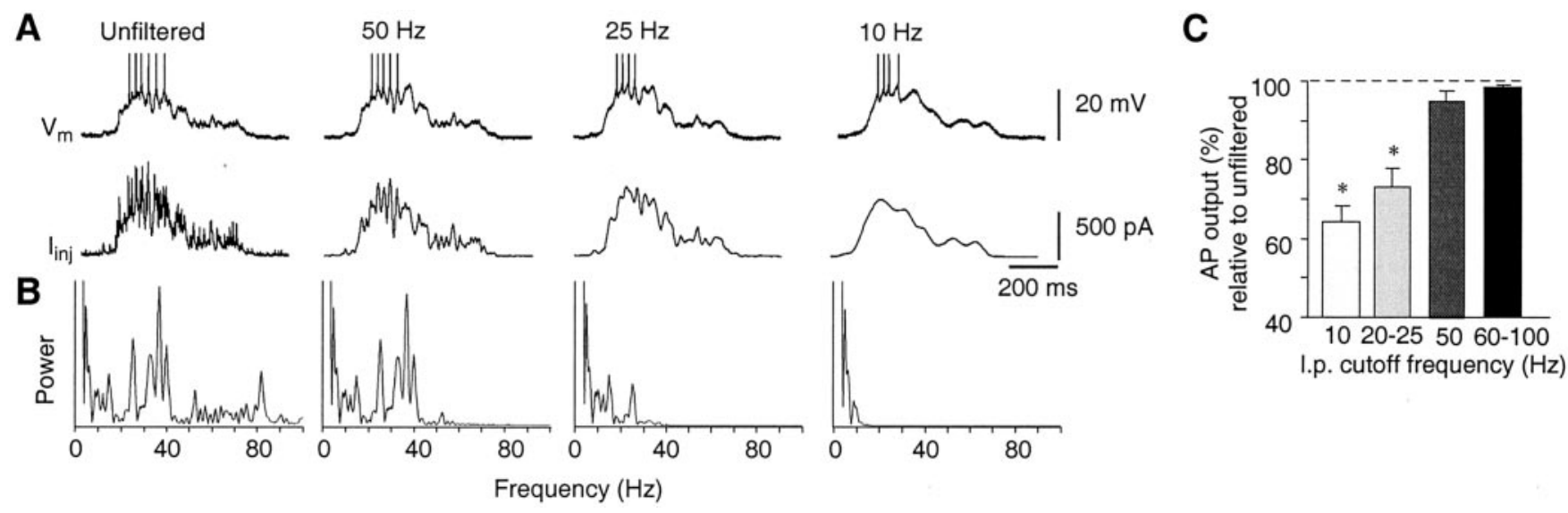

Figure 5. Removal of oscillations decreases efficacy of currents. $A$, Membrane potential responses of a PMN (top) to the same synaptic current waveform (bottom) injected before (unfiltered) and after low-pass filtering with cut-off frequencies set at 50,25 , and $10 \mathrm{~Hz}$ (action potentials are truncated). $B$, Power spectra corresponding to the waveforms shown in $A$ illustrate the effects of filtering on the power-frequency relationship. C, The number of action potentials evoked by stimulation of PMNs $(n=12)$ with synaptic current waveforms filtered at a variety of low-pass cut-off frequencies compared relative to the number of action potentials evoked by the unfiltered waveform (dashed line at 100\%).

arrays for sensory perception. Oscillations in this range are also prominent features of activity in the system controlling breathing. They are present in the inspiratory activities of medullary neurons underlying respiratory rhythm and pattern generation and in respiratory motoneurons, nerves, and muscles in all mammals studied (Cohen et al., 1997; Funk and Parkis, 2002). However, the function of inspiratory oscillations is not known. Here, we provide evidence for two important consequences of these oscillations for MN behavior: they control the timing of action potentials and increase input-output efficiency.

\section{Critique of method}

Simulated current waveforms have proven useful for exploring the relationship between current transients and firing probability and highlight the importance for firing behavior and action potential timing of dynamic changes in membrane potential (Mainen and Sejnowski, 1995; Nowak et al., 1997; Tang et al., 1997; Stevens and Zador, 1998; Volgushev et al., 1998; Rekling et al., 2000b; Beierholm et al., 2001; Powers and Binder, 2001), particularly the rise time and amplitude of EPSPs (Türker and Powers, 1999, 2002). To determine whether these effects are seen under more natural conditions of synaptic input, we reinjected endogenous inspiratory currents recorded from the same MNs. These somatic currents are relevant for functional investigations because they result from the membrane integration of behaviorally meaningful endogenous inputs arriving at synapses distributed over the somatodendritic membrane.

An important technical consideration is that currents recorded under voltage clamp may be greater than currents that would normally reach the soma. Under physiological conditions, i.e., no voltage clamp, synaptically evoked membrane depolarization reduces the driving force and magnitude of the non-NMDA receptor-mediated component of the synaptic current. The magnitude of this depolarization-mediated reduction in the nonNMDA component of the inspiratory current, however, will be partially offset by increased NMDA-mediated current (Liu and Feldman, 1992; Cook and Johnston, 1999). Increased membrane conductance associated with activation of glutamatergic inspiratory synapses (Liu et al., 1990) could also attenuate current transfer to the soma under physiological conditions. Reduction of this attenuation under voltage clamp could result in our recording a larger than normal synaptic current. In addition, when currents are reinjected during expiration, membrane conductance may be lower than during inspiration because of the lack of glutamatergic input. If any of these factors played a significant role in our experiments, reinjected currents would have produced larger membrane depolarizations than spontaneous inspiratory inputs. This did not appear to be the case. As discussed previously (Parkis et al., 2000) and in a representative sample of PMNs in the present study, the membrane depolarization evoked by reinjected synaptic currents was not significantly greater $(10 \%)$ than that evoked by spontaneous synaptic inputs. Thus, inspiratory currents recorded at the soma are similar to those reaching the soma under normal conditions.

\section{Functional significance}

Endogenous oscillatory inputs increase the efficiency with which MNs transduce input into output. This is consistent with responses of neocortical neurons to fluctuating inputs simulated by adding Gaussian noise to square-wave pulses (Tang et al., 1997). Thus, by driving PMNs with oscillatory synaptic currents, neuronal synchrony within respiratory motor networks can achieve the same output with less input. The biophysical basis of this efficiency gain extends beyond the benefits of simply summing coincident EPSCs to briefly drive the membrane above threshold. Action potentials coincided with oscillation peaks even when the underlying envelope of synaptic drive maintained membrane potential continuously above the repetitive firing threshold (as determined by square-wave inputs) (Fig. 3, compare $A, B$ ). Because action potential threshold decreases (Schlue et al., 1974; Dai et al., 2000) and firing probability increases (Türker and Powers, 1999) with increased rate of depolarization in MNs, coincidence of synaptic inputs functionally amplifies their effect by causing more rapid membrane depolarization (Azouz and Gray, 2000).

From a functional perspective, i.e., motoneuronal discharge producing muscle contraction, oscillatory inputs to MNs mean that generation of a given muscle force will require fewer action potentials from the premotoneuron pool. Similar efficiency gains throughout the respiratory network would reduce the metabolic cost of respiratory rhythm generation and drive transmission (i.e., fewer presynaptic action potentials will be required to evoke a postsynaptic action potential).

Consistent with experiments using simulated current transients (Mainen and Sejnowski, 1995) or oscillatory inputs (Tang 
et al., 1997; Volgushev et al., 1998), data in cortical neurons (Nowak et al., 1997) and our data in MNs demonstrate that endogenous oscillations in synaptic input strongly influence the timing of action potentials. Action potential timing may be profoundly important within higher neuronal networks by providing an additional coding dimension for information processing and the binding together of spatially distributed neuronal populations. At the level of $\mathrm{MN}$ and muscle behavior, the functional consequences of oscillations and their influence on action potential timing are threefold.

(1) Oscillations constrain the mechanisms by which neuromodulators affect MN activity. Potentiation of instantaneous firing frequency is a major effect of neuromodulators on $\mathrm{MN}$ responses to steady-state inputs. However, rather than increasing firing frequency of individual PMNs, which would have limited value when $\mathrm{MNs}$ are already firing at frequencies close to the fusion frequency of the muscle fibers they innervate (at and above fusion frequency, additional increases in MN firing frequency produce relatively smaller increments in force), the impact of neuromodulators on MNs driven by oscillating inputs will be achieved through recruitment of quiescent MNs and prolongation of firing duration. This conclusion is consistent with the observed PE-mediated potentiation of C4 (Fig. 4) and hypoglossal nerve inspiratory output (Selvaratnam et al., 1998). Modulators could increase discharge frequency during the active period (Lalley, 1986) if subthreshold oscillations interspersed between suprathreshold oscillations became suprathreshold (Tang et al., 1997) or if multiple rather than single spikes arose from each oscillatory peak. However, these mechanisms were not significant contributors under our experimental conditions. Firing frequency did not increase significantly after PE in any PMN driven by inspiratory waveforms or spontaneous inspiratory inputs. That these principles govern input-output processing of PMNs in general is supported by the observation that substance $\mathrm{P}$, like $\mathrm{PE}$, increases firing frequency of PMNs in response to squarewave but not inspiratory synaptic currents (Ptak et al., 2000). Thus, oscillations may serve to maintain MN firing frequency within an optimal range, reducing the variability in discharge frequency that might otherwise occur with the fluctuations in neuromodulator levels, and MN excitability, which accompany changes in activity (transitions from rest to exercise), stress (Stanford, 1995), and arousal state (Aston-Jones and Bloom, 1981).

Implicit in our data is that a change in $\mathrm{MN}$ firing frequency requires a change in the oscillation frequency. Indeed, in contrast to neuromodulatory inputs, some mechanosensory and chemosensory inputs are associated with small but significant increases in both oscillation frequency and firing frequency (Funk and Parkis, 2002).

(2) Oscillations impact the development of muscle force. The pattern of MN output, not simply the average frequency, determines muscle force (van Lunteren and Sankey, 2000). Thus, although efficient muscle activation is primarily attributed to the matching of MN firing properties and the contractile properties of their innervated muscle fibers, respiratory oscillations must also contribute. By controlling action potential timing, oscillations may increase the efficiency of diaphragmatic contraction by ensuring that muscle fibers are activated within an optimal range of frequencies. Oscillations can also prevent excessive PMN activation and the disastrous consequences of phrenic motoneuronal accommodation or diaphragmatic fatigue causing respiratory failure, by limiting the maximal instantaneous firing frequency. Perhaps for this reason (a) peaks in the power spectra of inspiratory inputs in neonatal rat PMNs (Liu et al., 1990; Parkis et al.,
1998) are similar to the fusion frequency of neonatal diaphragm (Martin-Caraballo et al., 2000), (b) the rate of diaphragmatic fatigue development increases significantly with motor unit stimulation frequencies $>60 \mathrm{~Hz}$ (Martin-Caraballo et al., 2000).

Control of action potential timing by the oscillations also implies that, for MNs receiving consistent oscillatory inputs, increases in muscle force are primarily achieved through recruitment of quiescent MNs. This contrasts with the convention that increases in firing frequency and recruitment both contribute.

(3) PMNs fire synchronously, as evidenced by common peaks in the power spectra of population (Smith and Denny, 1990) and unit activities, as well as peaks in the coherence spectra between phrenic nerve and PMN activities (Christakos et al., 1991). Shortterm synchrony, the tendency for some motor units to discharge within a few milliseconds of each other, occurs in many skeletal muscles (Sears and Stagg, 1976; Kirkwood et al., 1982; Datta, 1990; Farmer, 1998; Baker et al., 1999) and has been attributed to common input from synchronization of discharge in presynaptic fibers. Common inputs can also synchronize MN output (Binder and Powers, 2001; Türker and Powers, 2001, 2002), especially those composed of relatively few, large EPSPs (Halliday, 2000; Türker and Powers, 2002). Thus, the degree of synchrony between PMNs is likely to be higher than for many MN pools because PMNs are driven with large-amplitude, oscillatory patterns of synaptic input (Cohen et al., 1997).

Functionally, synchronous MN output may enhance intramuscular force transmission (Murthy and Fetz, 1994; Baker et al., 1999). Whether diaphragmatic muscle fibers are arranged exclusively in parallel, as in rat and rabbit (Gordon et al., 1989), or also in series, as in cat and dog (Gordon et al., 1989), tension is not simply delivered axially along a fiber. Some tension is transmitted through neighboring or serially arranged fibers and the extracellular matrix. Thus, during submaximal contractions typical of the diaphragm, delivery of force by any given motor unit will vary dynamically with the activity patterns of its neighbors or serial partners (Sheard et al., 2002). The problem this presents for generating smooth, consistent gradations of force could be solved by organizing premotor networks to ensure that anatomically coupled motor units are recruited synchronously as "functional units" (Sheard et al., 2002). Oscillations in inspiratory drive could serve this function. At present, however, whether the spatial relationship of endogenously coactive motor units is consistent with existence of such functional units is not known. The degree of synchronization that ultimately occurs between MNs may be task specific and reflect a balance between competing requirements for fine motor control, which decreases with increasing synchronization (Yao et al., 2000), and efficient contraction, which increases with synchronization (Baker et al., 1999). Because breathing is less dependent than other behaviors on the ability to produce fine gradations in force but requires instead that muscles remain rhythmically active throughout life, activation patterns may have evolved to favor efficiency.

\section{References}

Aston-Jones G, Bloom FE (1981) Activity of norepinephrine-containing locus coeruleus neurons in behaving rats anticipates fluctuations in the sleep-waking cycle. J Neurosci 1:876-886.

Azouz R, Gray CM (2000) Dynamic spike threshold reveals a mechanism for synaptic coincidence detection in cortical neurons in vivo. Proc Natl Acad Sci USA 97:8110-8115.

Baker SN, Kilner JM, Pinches EM, Lemon RN (1999) The role of synchrony and oscillations in the motor output. Exp Brain Res 128:109-117.

Beierholm U, Nielsen CD, Ryge J, Alstrom P, Kiehn O (2001) Characteriza- 
tion of reliability of spike timing in spinal interneurons during oscillating inputs. J Neurophysiol 86:1858-1868.

Binder MD, Powers RK (2001) Relationship between simulated common synaptic input and discharge synchrony in cat spinal motoneurons. J Neurophysiol 86:2266-2275.

Binder MD, Heckman CJ, Powers RK (1996) The physiological control of motoneuron activity. In: Handbook of physiology. Sect 12, Exercise: regulation and integration of multiple systems (Rowell L, Shepherd J, eds), pp 3-53. New York: Oxford UP.

Christakos CN, Cohen MI, Barnhardt R, Shaw CF (1991) Fast rhythms in phrenic motoneuron and nerve discharges. J Neurophysiol 66:674-687.

Cohen MI, Gootman PM (1969) Spontaneous and evoked oscillations in respiratory and sympathetic discharge. Brain Res 16:265-268.

Cohen MI, See WR, Christakos CN, Sica AL (1987) High-frequency and medium-frequency components of different inspiratory nerve discharges and their modification by various inputs. Brain Res 417:148-152.

Cohen MI, Huang W-X, See WR, Yu Q, Christakos CN (1997) Fast rhythms in respiratory neural activities. In: Neural control of the respiratory muscles, pp 159-169. Boca Raton, FL: CRC.

Cook EP, Johnston D (1999) Voltage-dependent properties of dendrites that eliminate location-dependent variability of synaptic input. J Neurophysiol 81:535-543.

Dai Y, Jones KE, Fedirchuk B, Jordan LM (2000) Effects of voltage trajectory on action potential voltage threshold in simulations of cat spinal motoneurons. Neurocomputing 32-33:105-111.

Datta AK (1990) Synchronization of motor unit activity during voluntary contraction in man. J Physiol (Lond) 422:397-419.

Engel AK, Fries P, Konig P, Brecht M, Singer W (1999) Temporal binding, binocular rivalry, and consciousness. Conscious Cogn 8:128-151.

Farmer SF (1998) Rhythmicity, synchronization and binding in human and primate motor systems. J Physiol (Lond) 509:3-14.

Fetz EE, Chen D, Murthy VN, Matsumura M (2000) Synaptic interactions mediating synchrony and oscillations in primate sensorimotor cortex. J Physiol (Paris) 94:323-331.

Funk GD, Parkis MA (2002) High frequency oscillations in respiratory networks: functionally significant or phenomenological? Respir Physiol Neurobiol 131:101-120.

Gordon DC, Hammond CG, Fisher JT, Richmond FJ (1989) Muscle-fiber architecture, innervation, and histochemistry in the diaphragm of the cat. J Morphol 201:131-143.

Gray CM (1994) Synchronous oscillations in neuronal systems: mechanisms and functions. J Comput Neurosci 1:11-38.

Halliday DM (2000) Weak, stochastic temporal correlation of large-scale synaptic input is a major determinant of neuronal bandwidth. Neural Comput 12:693-707.

Kirkwood PA, Sears TA, Tuck DL, Westgaard RH (1982) Variations in the time course of the synchronization of intercostal motoneurones in the cat. J Physiol (Lond) 327:105-135.

Konig P, Engel AK (1995) Correlated firing in sensory-motor systems. Curr Opin Neurobiol 5:511-519.

Lalley PM (1986) Serotoninergic and non-serotoninergic responses of phrenic motoneurones to raphe stimulation in the cat. J Physiol (Lond) 380:373-385.

Liu G, Feldman JL (1992) Bulbospinal transmission of respiratory drive to phrenic motoneurons. In: Respiratory control: central and peripheral mechanisms (Speck DF, Dekin MS, Revelette WR, Frazier DT, eds), pp 47-51. Lexington, KY: University Press of Kentucky.

Liu G, Feldman JL, Smith JC (1990) Excitatory amino acid-mediated transmission of inspiratory drive to phrenic motoneurons. J Neurophysiol 64:423-436.

Mainen ZF, Sejnowski TJ (1995) Reliability of spike timing in neocortical neurons. Science 268:1503-1506.

Martin-Caraballo M, Campagnaro PA, Gao Y, Greer JJ (2000) Contractile and fatigue properties of the rat diaphragm musculature during the perinatal period. J Appl Physiol 88:573-580.

Murthy VN, Fetz EE (1994) Effects of input synchrony on the firing rate of a three conductance cortical neuron model. Neural Comput 6:1111-1126.

Nakazawa K, Granata AR, Cohen MI (2000) Synchronized fast rhythms in inspiratory and expiratory nerve discharges during fictive vocalization. J Neurophysiol 83:1415-1425.
Nowak LG, Sanchez-Vives MV, McCormick DA (1997) Influence of low and high frequency inputs on spike timing in visual cortical neurons. Cereb Cortex 7:487-501.

Parkis MA, Bayliss DA, Berger AJ (1995) Actions of norepinephrine on rat hypoglossal motoneurons. J Neurophysiol 74:1911-1919.

Parkis MA, Robinson DM, Funk GD (2000) A method for activating neurons using endogenous synaptic waveforms. J Neurosci Methods 96:77-85.

Parkis MA, Dong X-W, Feldman JL, Funk GD (1999) Concurrent inhibition and excitation of phrenic motoneurons during inspiration: phasespecific control of excitability. J Neurosci 19:2368-2380.

Parkis MA, Dong X-W, Feldman JL, Robinson DM, Funk GD (1998) The $20-50 \mathrm{~Hz}$ bandwidth of inspiratory synaptic drive currents enhances action potential (AP) output from phrenic motoneurons (PMNs). Soc Neurosci Abstr 24:913.

Powers RK, Binder MD (2001) Input-output functions of mammalian motoneurons. Rev Physiol Biochem Pharmacol 143:137-263.

Ptak K, Konrad M, Di Pasquale E, Tell F, Hilaire G, Monteau R (2000) Cellular and synaptic effect of substance P on neonatal phrenic motoneurons. Eur J Neurosci 12:126-138.

Rekling J, Funk GD, Bayliss D, Dong X, Feldman JL (2000a) Synaptic control of motoneuronal excitability. Physiol Rev 80:767-852.

Rekling JC, Shao XM, Feldman JL (2000b) Electrical coupling and excitatory synaptic transmission between rhythmogenic respiratory neurons in the preBotzinger complex. J Neurosci 20:RC113(1-5).

Schlue WR, Richter DW, Mauritz KH, Nacimiento AC (1974) Responses of cat spinal motoneuron somata and axons to linearly rising currents. J Neurophysiol 37:303-309.

Sears TA, Stagg D (1976) Short-term synchronization of intercostal motoneurone activity. J Physiol (London) 263:357-381.

Selvaratnam SR, Parkis MA, Funk GD (1998) Developmental modulation of mouse hypoglossal nerve inspiratory output in vitro by noradrenergic receptor agonists. Brain Res 805:104-115.

Sheard P, Paul A, Duxson M (2002) Intramuscular force transmission. In: Advances in experimental medicine and biology, Vol 508, Sensori-motor control (Gandevia S, Proske U, Stuart D, eds), pp 495-499. New York: Kluwer Academic/Plenum.

Smith A, Denny M (1990) High-frequency oscillations as indicators of neural control mechanisms in human respiration, mastication, and speech. J Neurophysiol 63:745-758.

Smith JC, Feldman JL (1987) In vitro brainstem-spinal cord preparations for study of motor systems for mammalian respiration and locomotion. J Neurosci Methods 21:321-333.

Stanford SC (1995) Central noradrenergic neurones and stress. Pharmacol Ther 68:297-342.

Stevens CF, Zador AM (1998) Input synchrony and the irregular firing of cortical neurons. Nat Neurosci 1:210-217.

Tang AC, Bartels AM, Sejnowski TJ (1997) Effects of cholinergic modulation on responses of neocortical neurons to fluctuating input. Cereb Cortex 7:502-509.

Tarasiuk A, Sica AL (1997) Spectral features of central pattern generation in the in vitro brain stem spinal cord preparation of the newborn rat. Brain Res Bull 42:105-110.

Türker KS, Powers RK (1999) Effects of large excitatory and inhibitory inputs on motoneuron discharge rate and probability. J Neurophysiol 82:829-840.

Türker KS, Powers RK (2001) Effects of common excitatory and inhibitory inputs on motoneuron synchronization. J Neurophysiol 86:2807-2822.

Türker KS, Powers RK (2002) The effects of common input characteristics and discharge rate on synchronization in rat hypoglossal motoneurones. J Physiol (Lond) 541:245-260.

van Lunteren E, Sankey CB (2000) Catchlike property of rat diaphragm: subsequent train frequency effects in variable-train stimulation. J Appl Physiol 88:586-598.

Volgushev M, Chistiakova M, Singer W (1998) Modification of discharge patterns of neocortical neurons by induced oscillations of the membrane potential. Neuroscience 83:15-25.

Yao W, Fuglevand RJ, Enoka RM (2000) Motor-unit synchronization increases EMG amplitude and decreases force steadiness of simulated contractions. J Neurophysiol 83:441-452. 